\title{
Intrinsic structural distortion and superexchange interaction in the orthorhombic rare-earth perovskites $\mathrm{RCrO}_{3}$
}

\author{
J.-S. Zhou, ${ }^{1, *}$ J. A. Alonso, ${ }^{2}$ V. Pomjakushin, ${ }^{3}$ J. B. Goodenough, ${ }^{1}$ Y. Ren, ${ }^{4}$ J.-Q. Yan, ${ }^{5}$ and J.-G. Cheng ${ }^{1}$ \\ ${ }^{1}$ Texas Materials Institute, University of Texas at Austin, Austin, Texas 78712, USA \\ ${ }^{2}$ Instituto de Ciencia de Materiales de Madrid, CSIC, Cantoblanco, E-28049 Madrid, Spain \\ ${ }^{3}$ Laboratory for Neutron Scattering, Paul Scherrer Institut, ETH Zurich, CH-5232 Villigen PSI, Switzerland \\ ${ }^{4}$ Advanced Photon Source, Argonne National Laboratory, Argonne, Illinois 60439, USA \\ ${ }^{5}$ Division of Materials Science and Engineering, Ames Laboratory, Ames, Iowa 50011, USA
}

(Received 13 May 2010; published 18 June 2010)

\begin{abstract}
High-resolution neutron powder diffraction has been applied to determine the structural evolution in the orthorhombic perovskite $R \mathrm{CrO}_{3}$ family. The structural distortions observed have been found to be closely related to the dramatic variation in the magnetic ordering temperature $T_{\mathrm{N}}$. In addition to the reduction in the orbital overlap integral that can account for the change in $T_{\mathrm{N}}$ in the $R \mathrm{FeO}_{3}$ family, the effect of $t$-e hybridization due to the structural distortions are responsible for the dramatic change in $T_{\mathrm{N}}$ across the $R \mathrm{CrO}_{3}$ family.
\end{abstract}

DOI: 10.1103/PhysRevB.81.214115

\section{INTRODUCTION}

The magnetic properties of $\mathrm{RCrO}_{3}$ are extensively reported in three consecutive reviews of perovskite oxides that cover a huge number of studies over a half century. ${ }^{1}$ The magnetic structure of the orthochromites was also wellstudied during the boom of neutron diffraction in 1960s1970s. All kinds of magnetic structure in the orthorhombic perovskites can be well-described in connection with the crystal symmetry under the notation developed by Bertaut. ${ }^{2}$ In most cases, the collinearly ordered spins are along the $a$ or $c$ axis of the Pbnm cell. However, the coupling between the magnetic moment on the rare earth and the spins on $\mathrm{Cr}^{3+}$ is responsible for an easy-axis rotation below $T_{\mathrm{N}}$ in some orthochromites. ${ }^{3}$ Since the $T_{\mathrm{N}}$ of $R \mathrm{CrO}_{3}$ changes monotonically from $\mathrm{Lu}$ to $\mathrm{La}$ independent of the rare-earth moment, the $R$-O-Cr coupling appears to have negligible influence on the $\mathrm{Cr}-\mathrm{O}-\mathrm{Cr}$ coupling. It has been widely accepted that the $M-\mathrm{O}-M$ bond angle $(M=$ transition metal), which is reduced from $180^{\circ}$ due to the cooperative octahedral-site rotations in the orthorhombic perovskite, is a major factor entering the perturbation formula $J \sim b^{2}$ / $U$ of the $M-\mathrm{O}-M$ superexchange interaction; $b$ is the orbital overlap integral over the $M-\mathrm{O}-M$ bond and $U$ is the on-site Coulomb energy. This assumption has been shown to work properly in $\mathrm{RFeO}_{3}{ }^{4}$ In contrast, all Jahn-Teller (JT) active systems, such as $R \mathrm{TiO}_{3},{ }^{5,6} R \mathrm{VO}_{3},{ }^{7,8}$ and $R \mathrm{MnO}_{3},{ }^{9}$ do not show a clear connection between the magnetic transition temperatures and the $M-\mathrm{O}-M$ bond angle. Instead, they exhibit transitions between different types of spin orderings as the rare earth $R$ changes from $\mathrm{La}$ to $\mathrm{Lu}$. As in $R \mathrm{FeO}_{3}$, orbital ordering is not an issue in $R \mathrm{CrO}_{3}$; both families exhibit the type- $G$ spin ordering. Given the same superexchange coupling parameter $J$ in the formula $k_{\mathrm{B}} T_{\mathrm{N}}=4 S(S+1) \mathrm{J}$, a huge difference between $T_{\mathrm{N}}=760 \mathrm{~K}$ in $\mathrm{LaFeO}_{3}$ and $T_{\mathrm{N}}=298 \mathrm{~K}$ in $\mathrm{LaCrO}_{3}$ can be well accounted for by the total spin $S=5 / 2$ for $\mathrm{Fe}^{3+}: t^{3} e^{2}$ and $S=3 / 2$ for $\mathrm{Cr}: t^{3} e^{0}$. However, a dramatic change in $T_{\mathrm{N}}$ from $\mathrm{LaCrO}_{3}$ to $\mathrm{LuCrO}_{3}$ appears troublesome for applying the same overlap integral reduction as in $R \mathrm{FeO}_{3}$. Due to incomplete structural data, no attempt has been made so far to answer whether the $M-\mathrm{O}-M$ bond-angle evolution over $R^{3+}$ ion can justify the
PACS number(s): 61.66.Fn, 61.05.F-, 75.10.Hk, 75.30.Et

change in Néel temperature in the $\mathrm{RCrO}_{3}$ family. In this paper, we report results of high-resolution neutron diffraction on all $R \mathrm{CrO}_{3}$ members except a few neutron-absorbing rare earths. By comparison with the structural work of the $R \mathrm{FeO}_{3}$ family in the literature, we are able to identify the local structural distortion that is intrinsic to the orthorhombic perovskite. We have shown that the scenario of overlap integral versus $T_{\mathrm{N}}$, which has been assumed to be generally applicable in the superexchange interaction, is limited to some special cases like in $R \mathrm{FeO}_{3}$ where both $t$ and $e$ orbitals are half filled. The observed overall change in $T_{\mathrm{N}}$ across the $R \mathrm{CrO}_{3}$ family can be explained well only after the effect of $t-e$ hybridization due to the local site distortion and cooperative octahedral-site rotation is taken into account.

\section{EXPERIMENT}

Polycrystalline samples of $R \mathrm{CrO}_{3}$ were prepared by standard solid-state reactions. The mixtures of rare-earth oxides and chromium oxide in a stoichimetric ratio were sintered in air at $980-1450{ }^{\circ} \mathrm{C}$ with several intermediate grindings. We prepared about $7 \mathrm{~g}$ of powder sample for each member in the $R \mathrm{CrO}_{3}$ family to ensure high quality neutron-diffraction data. These materials were checked to be single phase by $\mathrm{x}$-ray powder diffraction (XRD). Neutron powder-diffraction (NPD) patterns were collected at the high-resolution powder diffractometer (HRPT) (Ref. 10) of the SINQ spallation source at the Paul Scherrer Institute, Villigen, Switzerland. The samples were packed in cylindrical vanadium holders of $6 \mathrm{~mm}$ diameter excepting the $\mathrm{DyCrO}_{3}$, which was contained in a double-walled sample holder to minimize neutron absorption. The patterns were collected at room temperature with a wavelength of $1.494 \AA$. In all cases the high-intensity mode was used; the collection time was about $4 \mathrm{~h}$ per diagram $\left(20 \mathrm{~h}\right.$ for $\left.\mathrm{DyCrO}_{3}\right)$. The NPD patterns were analyzed with the Rietveld method with the FULLPROF (Ref. 11) program. The magnetization measurements were carried out in a superconductor quantum interference device magnetometer (Quantum Design). All crystal-structure and magnetization data will be published separately. We present here our major results. 


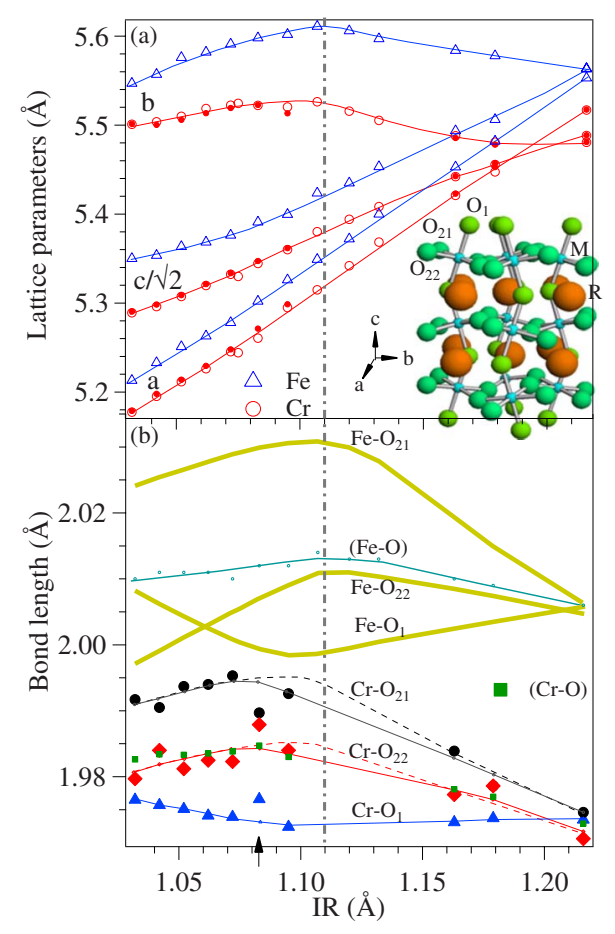

FIG. 1. (Color online) The IR dependences crossing all rareearth ions from $\mathrm{Lu}^{3+}$ to $\mathrm{La}^{3+}$ of (a) lattice parameters (solid symbols are from neutron diffraction) and (b) $M$-O bond lengths of $\mathrm{RCrO}_{3}$. Error bars are comparable to the size of symbols. The anomalous bond lengths of $\mathrm{DyCrO}_{3}$, shown by an arrow, are attributed to the Dy neutron absorption. The inset shows the structural model of the Pbnm perovskite.

\section{RESULTS AND DISCUSSION}

A precise knowledge of the crystal structure is always essential for interpretation of the physical properties. We first made a thorough analysis of the crystal structure of $R \mathrm{CrO}_{3}$ in comparison with that of $R \mathrm{FeO}_{3}$ since the latter is a model system in which the $T_{\mathrm{N}}$ variation can be well accounted for by the $M-\mathrm{O}-M$ bond angle. We start by analyzing the lattice parameters of $R \mathrm{CrO}_{3}$ and $R \mathrm{FeO}_{3}$ as a function of the ionic radius (IR) of the $R^{3+}$ ions, Fig. 1(a). The lattice parameters in both systems show the same trend as IR increases. Major features include (1) $b>c / \sqrt{2}>a$ for most $R$ as required by the crystal symmetry of the Pbnm space group provided that the octahedra are rigid and (2) the curve $b$ versus IR shows an anomaly near IR=1.11 $\AA$ in contrast to the straight line predicted by the software SPUDS (Ref. 12) for rigid octahedra. The anomaly in feature (2) is universal for all the orthorhombic perovskites ${ }^{13}$ as the $\mathrm{O}_{21}-M-\mathrm{O}_{22}$ bond angle shown in Fig. 2(a) starts to decrease from $90^{\circ}$ for IR $>1.11 \AA$. The resulting decrease in the rotation $b$ axis leads eventually to a $b<a$ as is seen in $\mathrm{LaCrO}_{3}$. As a general character of the Pbnm perovskites, the long $M$-O bond is always within the $a b$ plane. The $\mathrm{Fe}-\mathrm{O}_{21}$ and $\mathrm{Fe}-\mathrm{O}_{22}$ bond lengths reach their maximum near IR=1.11 $\AA$; at the same IR, the apical $\mathrm{Fe}-\mathrm{O}_{1}$ is located at a minimum. Although no data are available for the $R \mathrm{CrO}_{3}$ with the neutron absorbers $R=\mathrm{Sm}, \mathrm{Eu}$, and $\mathrm{Gd}$, we have good reasons to believe that bond lengths versus IR follow the dashed lines in Fig. 1(b) similar to that of $R \mathrm{FeO}_{3}$

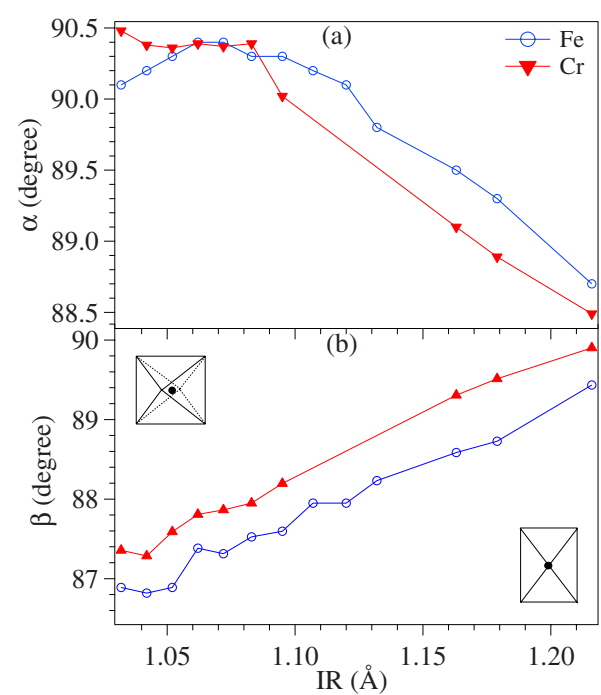

FIG. 2. (Color online) The $\operatorname{IR}\left(R^{3+}\right)$ dependence of (a) the $\mathrm{O}_{21}-M-\mathrm{O}_{22}$ bond angle $\alpha$ and (b) the angle $\beta$ between $M-\mathrm{O}_{1}$ bond and the $\mathrm{O}_{21}-M-\mathrm{O}_{22}$ plane. Insets: octahedron projected along the axis normal to the $\mathrm{O}_{21}-M-\mathrm{O}_{22}$ plane.

since they all share the same features of lattice parameters. The largest bond-length splitting in both systems occurs near $\mathrm{IR}=1.11 \AA$. The classic case of $R \mathrm{FeO}_{3}$ was accomplished by using $\mathrm{x}$-ray single-crystal diffraction ${ }^{14}$ in which the $\mathrm{Fe}-\mathrm{O}$ bond lengths were determined within large error bars. We have reproduced here in Fig. 1(b) the guide lines that go through scattered data points of the $\mathrm{Fe}-\mathrm{O}$ bond lengths versus IR in $R \mathrm{FeO}_{3} .{ }^{14}$ The results of our NPD data trace down the profile of $\mathrm{Cr}-\mathrm{O}$ bonds as a function of IR with high precision. Since there is no orbital order to have an influence on the magnetic interactions in these two JT-inactive systems, we focus on the superexchange interaction that is modulated by structural distortions.

We turn to the $\mathrm{Cr}-\mathrm{O}-\mathrm{Cr}$ bond angle, the so-called "superexchange angle" in the orthorhombic perovskites. As shown in Fig. 3(a), the averaged $\mathrm{Cr}-\mathrm{O}-\mathrm{Cr}$ bond angle $\theta$ is progressively reduced from $180^{\circ}$ as the IR decreases. As a matter of fact, the profile of $\theta$ versus IR is nearly identical in several orthorhombic perovskites $R M \mathrm{O}_{3}(M=\mathrm{Ti}, \mathrm{V}, \mathrm{Cr}, \mathrm{Mn}, \mathrm{Fe}, \mathrm{Ga})$. For a given $R, R \mathrm{CrO}_{3}$ has the smallest octahedral-site tilting angle among these perovskite families because of the relatively smaller bond-valence-sum radius of the $\mathrm{Cr}^{3+}$ ion and therefore larger geometric tolerance factor $t$. Without a rigorous proof so far, the empirical relationship between the superexchange coupling $J$ and the superexchange angle for the $R \mathrm{FeO}_{3}$ family has undergone an evolution from $J$ $\sim \cos \theta$ (Ref. 15) to $J \sim \cos ^{2} \theta$ (Ref. 16) and finally to $J$ $\sim \cos ^{4}(\omega / 2) / d^{7}$ (Ref. 4), where $d$ denotes the $M$-O bond length and $\omega=180-\theta$. As shown in Fig. 1(b), $d$ is essentially independent of IR for $R \mathrm{FeO}_{3}$ and can be taken as an averaged bond length. In order to make a quantitative comparison of the calculated $T_{\mathrm{N}}$ based on the structural data and the observed $T_{\mathrm{N}}$, we have normalized $T_{\mathrm{N}}$ by that of $\mathrm{La} M \mathrm{O}_{3}$. The calculation based on the formula $J \sim \cos ^{4}(\omega / 2) / d^{7}$ can indeed produce the essential feature of the observed $T_{\mathrm{N}}$ versus IR for the $R \mathrm{FeO}_{3}$ family in Fig. 3(b). However, this angle dependence can only account for about $30 \%$ of the overall 


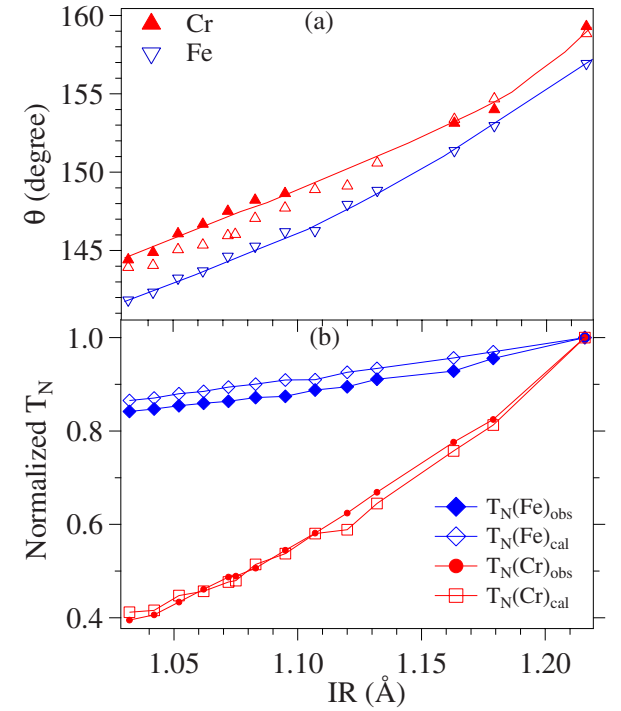

FIG. 3. (Color online) The $\operatorname{IR}\left(R^{3+}\right)$ dependences of (a) the averaged $M-\mathrm{O}-M$ bond angle $\theta$ (hollow symbols are from XRD) and (b) normalized $T_{\mathrm{N}}$ of $R \mathrm{FeO}_{3}$ and $R \mathrm{CrO}_{3}$ by $T_{\mathrm{N}}=740 \mathrm{~K}$ for $\mathrm{LaFeO}_{3}$ and $289 \mathrm{~K}$ for $\mathrm{LaCrO}_{3}$, respectively. $T_{\mathrm{N}}$ of $R \mathrm{FeO}_{3}$ are after Ref. 15.

change in $T_{\mathrm{N}}$ across the $R \mathrm{CrO}_{3}$ family. Some important spinspin interaction term in addition to the change in the superexchange angle is missing in our understanding of the $\mathrm{RCrO}_{3}$ family.

What has been ignored is the hybridization between intersite $t$ - $e$ orbitals which are orthogonal for a $180^{\circ} M-\mathrm{O}-M$ bond. As illustrated in Fig. 4, octahedral-site tilting not only reduces the $t$ orbital overlap integral considered but also introduces the orbital overlap between the $\pi$ and the $\sigma$ bonds. The influence on the overall exchange interaction of the intersite $t-e$ orbital hybridization is sharply different for $\mathrm{Fe}^{3+}: t^{3} e^{2}$ versus $\mathrm{Cr}: t^{3} e^{0}$. The superexchange interaction between two adjacent transition-metal ions is delivered by a virtual charge transfer (VCT). Figure 5 illustrates the case for

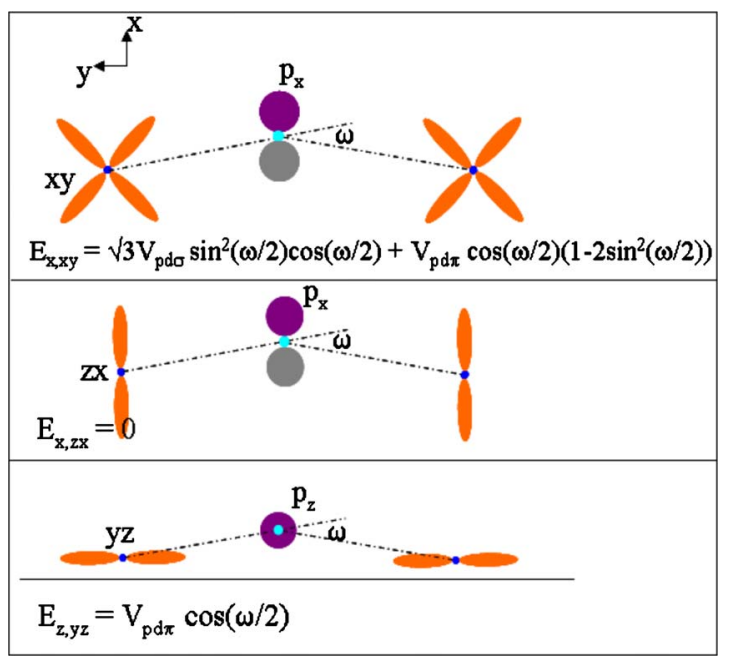

FIG. 4. (Color online) Schematic diagram of orbital overlap integrals. These are the examples of 15 two-center orbital overlap integrals. Formulas inside diagram show the angle dependence of the overlap integral. See text for the bond-length dependence $V_{p d}$.
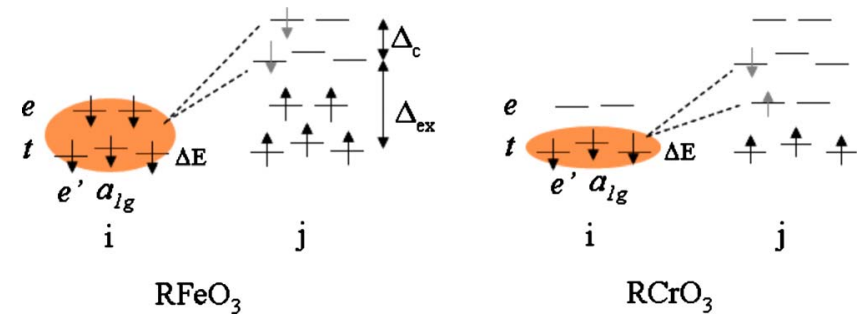

FIG. 5. (Color online) Schematic diagram of the hybridization effect on the virtual charge transfer for the superexchange interaction in perovskites $R \mathrm{CrO}_{3}$ and $\mathrm{RFeO}_{3}$. The VCT occurs for all occupied spin states; only those for the representative magnetic couplings are shown in the figure. $\Delta_{\mathrm{c}}$ stands for the crystal-field splitting, $\Delta_{\mathrm{ex}}$ is the exchange splitting, $\Delta E=E_{\mathrm{a} 1 \mathrm{~g}}-E_{\mathrm{e}}$.

the $\mathrm{Fe}^{3+}$ ions. The superexchange interactions over the halffilled $\pi$ bond $t^{3}-\mathrm{O}-t^{3}$ and the half-filled $\sigma$ bond $e^{2}-\mathrm{O}-e^{2}$ are all antiferromagnetic (AF) according to the superexchange rules. The $t$ - $e$ hybridization does not bring in any new component to the overall superexchange interaction in this case. The reduction in the $\sigma$ bond overlap integral $b^{\sigma}$ due to octahedral-site rotation appears not to be compensated by a small gain of the overlap integral through the intersite $t$ - $e$ hybridization. Therefore, the angular dependence of $J$ $\sim \cos ^{4}(\omega / 2) / d^{7}$, which considers only the $\sigma$ bond, is still a good approximation. In the case of $R \mathrm{CrO}_{3}$, however, only the $t$ orbitals are half filled. A $t-e$ hybridization similar to that in $R \mathrm{FeO}_{3}$ introduces a VCT to the empty $e$ component of the hybridized orbital at site $j$, i.e., $t^{3}-\mathrm{O}-e^{0}$, which leads to a ferromagnetic coupling $J_{\mathrm{hb}}^{\sigma}$ according to the superexchange rules. The cartoon for $\mathrm{RCrO}_{3}$ in Fig. 5 shows the $t$-e hybridization that leads to the ferromagnetic VCT to the empty $e$ orbital on site $j$ whereas the $t^{3}-\mathrm{O}-t^{3}$ transfer gives a dominant AF coupling $J^{\pi}$. The final superexchange interaction for the type $G$ antiferromagnetic ordering is $J=J^{\pi}-J_{\mathrm{hb}}^{\sigma}$. The progressively enhanced $J_{\mathrm{hb}}^{\sigma}$ becomes comparable to the angle- $\theta$-dependent $J^{\pi}$ as IR decreases. In order to check whether this argument can account quantitatively for the observed $T_{\mathrm{N}}$ versus IR, we have derived $J^{\pi}$ and $J_{\mathrm{hb}}^{\sigma}$ by including all possible two-center orbital overlap integrations between $\left(p_{x}, p_{y}, p_{z}\right)$ on $\mathrm{O}^{2-}$ and $\left(x y, y z, z x, x^{2}-y^{2}, z^{2}-r^{2}\right)$ on $\mathrm{Cr}^{3+}$ based on the Slater-Koster tables of interatomic matrix elements, ${ }^{17}$ which gives the following angle dependence for the orbital overlap integrals $b^{\pi}$ and $b_{\mathrm{hb}}^{\sigma}$

$$
\begin{gathered}
b_{\mathrm{hb}}^{\sigma}=[\cos (\omega / 2)+\sin (\omega / 2)]\{\sqrt{3} \cos (\omega / 2) \sin (\omega / 2) \\
\left.+\frac{\sqrt{3}}{2}\left[\cos ^{2}(\omega / 2)-\sin ^{2}(\omega / 2)\right]-\frac{1}{2}\right\} V_{p d \sigma}, \\
b^{\pi}=\left\{2[\sin (\omega / 2)+\cos (\omega / 2)]-4 \sin (\omega / 2) \cos ^{2}(\omega / 2)\right\} V_{p d \pi} .
\end{gathered}
$$

Therefore, the superexchange coupling $J$ can be expressed by

$$
J=J_{0}\left[\left(b^{\pi}\right)^{2}-\eta\left(b_{\mathrm{hb}}^{\sigma}\right)^{2}\right], \quad \eta=\frac{U+\Delta_{\mathrm{ex}}}{U+\Delta_{\mathrm{c}}}\left(\frac{V_{p d \sigma}}{V_{p d \pi}}\right)^{2} .
$$

$J_{0}$ here is a constant that determines the absolute value of $T_{\mathrm{N}}$ but it has no effect on the calculation of a normalized $T_{\mathrm{N}}$ in 
Fig. 3(b). A match between the observed $T_{\mathrm{N}}$ and the calculated value like that shown in Fig. 3(b) requires an $\eta=1.5$. That is a highly reasonable number since the bond-lengthdependent integral $V_{p d \sigma}$ is slightly larger than $V_{p d \pi}$ in oxides as has been shown by Harrison ${ }^{18}$ and $\Delta_{\mathrm{ex}}$ is slightly larger than $\Delta_{c}$ in octahedral-site $\mathrm{Cr}^{3+}$.

Another local site distortion is the angle $\beta$ between the apical $M-\mathrm{O}_{1}$ bond and the $\mathrm{O}_{21}-M-\mathrm{O}_{22}$ plane. As seen from Fig. 2(b), this angle is near $90^{\circ}$ for $\mathrm{LaFeO}_{3}$ but it is reduced monotonically as IR decreases. The same angle change can also be seen in $R \mathrm{CrO}_{3}$. Landron and Lepetit ${ }^{19}$ have shown that the intrasite hybridization between $t$ and $e$ orbitals is highly sensitive to the angle $\beta$ (they have defined the trigonal distortion of an octahedron by a different angle). In our case, the intrasite $t$ - $e$ hybridization vanishes for $\beta=90^{\circ}$. The component of $e$ orbital mixing into the $t$ orbital due to the trigonal distortion of the octahedral site is extremely small in our case but the intrasite hybridization splits the degenerated $t_{2}$ orbitals into $2 \times e^{\prime}$ and $a_{1 \mathrm{~g}}$. The intrasite hybridization strengthens the ferromagnetic coupling $J_{\mathrm{hb}}^{\sigma}=\left(\mathrm{b}_{\mathrm{hb}}^{\sigma}\right)^{2} /\left(U+\Delta_{\mathrm{c}}\right)$ by offsetting a $\Delta E=E_{\mathrm{e}^{\prime}}-E_{\mathrm{a} 1 \mathrm{~g}}$ in the energy difference $U$ $+\Delta_{\mathrm{c}}$ associated with this VCT.

The intersite $t$ - $e$ hybridization should influence physical properties in all orthorhombic perovskites. The $\mathrm{RCrO}_{3}$ family with $t^{3} e^{0}$ exemplifies clearly the effect. The same mechanism should also be applicable to another system with $t^{3} e^{0}$, for example, the perovskites $A \mathrm{Mn}^{4+} \mathrm{O}_{3} \quad(A=\mathrm{Ca}$, Sr, and $\mathrm{Sr}_{1-x} \mathrm{Ba}_{x}$ ) (Ref. 20) where $T_{\mathrm{N}}$ is found to be very sensitive, though less dramatically than that in $\mathrm{RCrO}_{3}$, to IR. However, other factors such as the size variance and the change in $A-O$ covalency have to be included in order to account for the curve of $T_{\mathrm{N}}$ versus IR quantitatively in the $A M^{4+} \mathrm{O}_{3}$ perovskites. In the JT-active family $R \mathrm{TiO}_{3}$, orbitals remain disordered in the paramagnetic phase. Fitting to a Curie-Weiss law of the paramagnetic susceptibility gives an antiferromagnetic coupling for the $R \mathrm{TiO}_{3}$ family with larger IR and a ferromagnetic coupling for those with smaller IR. The intrinsic local site distortions are certainly playing a role for the transition of the magnetic interaction by confining orbital fluctuations in such a way that the AF $t^{1}-\mathrm{O}-t^{1}$ coupling is preferred for the $R \mathrm{TiO}_{3}$ with large IR and the ferromagnetic $t^{1}$-O- $t^{0}$ for those with smaller IR. However, long-range magnetic order and orbital order occur at the same temperature, ${ }^{6}$ which indicates that orbital order is a prerequisite to longrange magnetic order. The increasing ferromagnetic $t^{1}-\mathrm{O}-e^{0}$ coupling as IR decreases contributes to the transition from the antiferromagnetic phase to the ferromagnetic phase shown in the phase diagram of the $R \mathrm{TiO}_{3}$ family. ${ }^{5} \mathrm{In}$ another JT-active family, $R \mathrm{VO}_{3}$, the orbital flipping transition in the magnetically ordered phase occurring for IR $\leq r_{\text {Dy }}$ cannot be explained only by the change in geometric tolerance factor $t$.
A careful comparison between the effects of hydrostatic pressure versus uniaxial pressure has clarified the effect to be due to the $t-e$ hybridization in these highly distorted perovskites. $^{21}$

\section{CONCLUSION}

The structural evolution of orthorhombic $R \mathrm{CrO}_{3}$ has been resolved by high-resolution neutron diffraction. A side-byside comparison with that of $R \mathrm{FeO}_{3}$ gives a solid proof that the following local structural distortions are intrinsic to orthorhombic perovskites $R M \mathrm{O}_{3}$ : (a) the longest $M-\mathrm{O}$ bond length is within the $\mathrm{O}_{21}-M-\mathrm{O}_{22}$ plane, (b) the bond-length splitting undergoes a maximum at a middle IR near $1.11 \AA$, (c) at the same IR the $\mathrm{O}_{21}-M-\mathrm{O}_{22}$ bond angle $\alpha$ starts to deviate from $90^{\circ}$, and (d) the angle $\beta$ between the apical $M-\mathrm{O}_{1}$ bond and the $\mathrm{O}_{21}-M-\mathrm{O}_{22}$ plane, which is near $90^{\circ}$ for $\mathrm{LaFeO}_{3}$ and $\mathrm{LaCrO}_{3}$, reduces monotonically as IR decreases. These intrinsic structural distortions are in addition to the cooperative octahedral-site rotations, widely called the $\mathrm{GdFeO}_{3}$-type distortion, and are essential elements to interpret physical properties in these perovskites. As a consequence of structural distortions that include the cooperative octahedral-site rotation and local distortions, the $t$ - $e$ hybridization is inevitable. For $R \mathrm{FeO}_{3}$, where the AF coupling consists of the superexchange interactions of the $t^{3}-\mathrm{O}-t^{3}$ and $e^{2}-\mathrm{O}-e^{2}$ orbitals, the hybridization does not bring any new component. Therefore the empirical angle dependence of the superexchange coupling $J$ remains a good approximation. The more dramatic change in $T_{\mathrm{N}}$ in the $R \mathrm{CrO}_{3}$ family highlights the importance of the $t-e$ hybridization effect. The ferromagnetic coupling through the superexchange interaction $t^{3}-\mathrm{O}-e^{0}$ is comparable to the antiferromagnetic coupling through the superexchange interaction $t^{3}-\mathrm{O}-t^{3}$. By considering all possible two-center orbital overlap integrals involved in the virtual charge transfer for the superexchange interaction in the orthorhombic perovskite structure, we are able to account quantitatively for the observed $T_{\mathrm{N}}$ versus IR in the $R \mathrm{CrO}_{3}$ family.

\section{ACKNOWLEDGMENTS}

This work was supported by NSF (Grant No. DMR 0904282) and the Robert A Welch Foundation (Grant No. F-1066), as well as the Spanish Ministry of Science and Innovation (Grant No. MAT2007-60536). The neutron diffraction performed at PSI, Switzerland was partially supported by the European Commission under the Seventh Framework Program through the "Research Infrastructures" action of the "Capacities" Program, Contract No. CP-CSA_INFRA-20081.1.1 Number 226507-NMI3'. J.S.Z. thanks W. Harrison for the insightful discussion. 
*jszhou@mail.utexas.edu

${ }^{1}$ J. B. Goodenough and J. M. Longo, Crystallographic and magnetic properties of perovskite and perovskite-related compounds, Landolt-Bornstein, New Series, Group III Vol. 4, Pt. A (Springer-Verlag, Berlin, 1970), p. 126; K. Enke, J. Fleischhauer, W. Gunber, P. Hansen, S. Nomura, W. Tolksdorf, G. Winkler, and U. Wolfmeier, Magnetic and other properties of oxides and related compounds, Landolt-Bornstein, New Series, Group III Vol. 12 (Springer-Verlag, Berlin, 1978), p. 368; Y. Endoh, K. Kakurai, A. K. Katori, M. S. Seehra, G. Srinivasan, and H. P. J. Wijn, Magnetic properties of non-metallic inorganic compounds based on transition elements, Subvolume f3, Perovskites II, Oxides with Corundum, Ilmenite and Amorphous Structures, Landolt-Bornstein, New Series, Group III Vol. 27 (Springer-Verlag, Berlin, 1994), p. 1.

${ }^{2}$ E. F. Bertaut, in Magnetism, edited by G. T. Rado and H. Suhl (Academic Press, New York, 1963), Vol. 3, p. 149.

${ }^{3}$ R. M. Hornreich, J. Magn. Magn. Mater. 7, 280 (1978).

${ }^{4}$ J.-S. Zhou and J. B. Goodenough, Phys. Rev. B 77, 132104 (2008).

${ }^{5}$ A. C. Komarek, H. Roth, M. Cwik, W.-D. Stein, J. Baier, M. Kriener, F. Bouree, T. Lorenz, and M. Braden, Phys. Rev. B 75, 224402 (2007).

${ }^{6}$ J.-G. Cheng, Y. Sui, J.-S. Zhou, J. B. Goodenough, and W. H. Su, Phys. Rev. Lett. 101, 087205 (2008).

${ }^{7}$ J.-S. Zhou, J. B. Goodenough, J.-Q. Yan, and Y. Ren, Phys. Rev. Lett. 99, 156401 (2007).

${ }^{8}$ J.-Q. Yan, J.-S. Zhou, J. B. Goodenough, Y. Ren, J.-G. Cheng, S. Chang, J. Zarestky, O. Garlea, A. Llobet, H. D. Zhou, Y. Sui, W. H. Su, and R. J. McQueeney, Phys. Rev. Lett. 99, 197201
(2007).

${ }^{9}$ J.-S. Zhou and J. B. Goodenough, Phys. Rev. Lett. 96, 247202 (2006).

${ }^{10}$ P. Fischer, G. Frey, M. Koch, M. Konnecke, V. Pomjakushin, J. Schefer, R. Thut, N. Schlumpf, R. Burge, U. Greuter, S. Bondt, and E. Berruyer, Physica B 276-278, 146 (2000).

${ }^{11}$ J. Rodriguez-Carvajal, Physica B 192, 55 (1993).

${ }^{12}$ M. W. Lufaso and P. M. Woodward, Acta Crystallogr., Sect. B: Struct. Sci. 57, 725 (2001).

${ }^{13}$ J.-S. Zhou and J. B. Goodenough, Phys. Rev. Lett. 94, 065501 (2005).

${ }^{14}$ M. Marezio and P. D. Dernier, Mater. Res. Bull. 6, 23 (1971); M. Marezio, J. P. Remeika, and P. D. Dernier, Acta Crystallogr., Sect. B: Struct. Crystallogr. Cryst. Chem. 26, 2008 (1970).

${ }^{15}$ D. Treves, M. Eibschutz, and P. Coppens, Phys. Lett. 18, 216 (1965); M. Eibschütz, and D. Treves, Phys. Rev. 156, 562 (1967).

${ }^{16}$ C. Boekema, F. van der Woude, and G. A. Sawatzky, Int. J. Magn. 3, 341 (1972).

${ }^{17}$ J. C. Slater and G. F. Koster, Phys. Rev. 94, 1498 (1954).

${ }^{18}$ W. A. Harrison, Elementary Electronic Structure (World Scientific, New Jersey, 2004), pp. 646, 693-703.

${ }^{19}$ S. Landron and M.-B. Lepetit, Phys. Rev. B 77, 125106 (2008).

${ }^{20}$ O. Chmaissem, B. Dabrowski, S. Kolesnik, J. Mais, D. E. Brown, R. Kruk, P. Prior, B. Pyles, and J. D. Jorgensen, Phys. Rev. B 64, 134412 (2001).

${ }^{21}$ J.-S. Zhou, J. B. Goodenough, J.-Q. Yan, J.-G. Cheng, K. Matsubayashi, Y. Uwatoko, and Y. Ren, Phys. Rev. B 80, 224422 (2009). 